\title{
Applied Grid Computing: Optimisation of Photonic Devices
}

\author{
Duan H Beckett ${ }^{1}$, Ben Hiett ${ }^{1}$, Ken S Thomas ${ }^{1}$, and Simon J Cox ${ }^{2}$ \\ ${ }^{1}$ Department of Electronics and Computer Science, University of Southampton, \\ SO17 \\ ${ }^{2}$ School of Engineering Sciences, University of Southampton, SO17 1BJ
}

\begin{abstract}
In this paper, we present an application of grid computing to solve an important industrial problem: that of optimising the band gap of photonic crystals, which are an important technology in future generation telecomms and sensing. The computational power grid enabled months of experimentation to be performed in a weekend. Of particular interest was the necessity to run jobs on both Linux and Windows resources.
\end{abstract}

\section{Introduction}

Applications such a sharp angle waveguides, wave division multiplexing, single mode lasers and sensors have motivated research into photonic crystals; which are nanostructured devices that filter light at particular frequencies. Photonic crystals are modeled by finding periodic solutions to Maxwell's equations [1], [2], [3]. A typical finite element analysis will comprise: mesh generation for the domain, selection of suitable basis functions and the solution of a generalised eigenvalue problem

$$
A(k) c=\lambda B c .
$$

The eigenvalue problem is solved for a large sample of quasi momentum vectors $k$ in the Brillouin zone, because the spectrum of the original problem in $R^{2}$ is the union of the spectrum of (1) over $k \in B$ [3]. The band gaps are the resolvent set of the original problem.

The absolute size of the band gap is not a meaningful measurement because there is no fundamental length scale in electromagnetics [4] and so we find the configuration of the rods that gives the largest gap-midgap ratio. This optimisation and the important role played by a computational grid is the aim of this paper.

The University of Southampton has invested in grid-enabled computational facilities. We used a Beowulf cluster [5] running Linux and an experimental Condor system [6] that uses Windows. One important factor was that our chosen meshing program Geompack [7]is only available for Windows, thus requiring a heterogeneous mix of operating systems in our grid. 


\section{Optimising Photonic Crystal Structures}

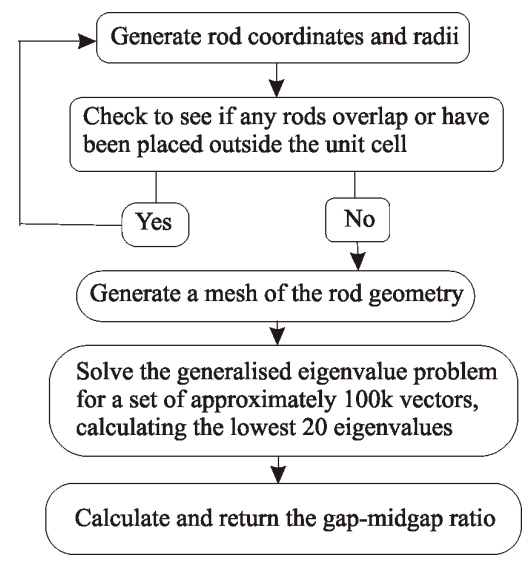

Fig. 1. Figure showing how the objective function for maximising the PC gap-midgap ratio is constructed.

A photonic crystal is a periodic configuration of air-filled rods in a substrate (for example Gallium Arsenide). The physical properties of a Photonic Crystal (PC) depend critically on the configuration of air rods in a substrate. A useful PC would be one such that the gap-midgap ratio [4] is very large. The objective in the optimisation is to maximize the gap-midgap ratio; the independent variables are the rod co-ordinates and the rod radii. The details of the computation are shown in Figure 1. Our approach is more direct than [8], [9], who only consider the TM mode and used a gradient method.

To investigate various $\mathrm{PC}$ geometries we performed a design of experiment which consisted of constructing several thousand random configurations of a number of rods in a unit cell. Two different unit cells were used: a square unit cell and a rhombic unit cell. We only report on the latter in this paper. The rhombic unit cell was varied from 1 to 10 rods, although only the radius was altered when a single rod was used as the position for a single rod is meaningless when the unit cell is tiled.

For the rhombic unit cell 100 meshes per rod radius and number of rods was generated for varying filling fractions of approximately $30 \%$ to $78 \%$. The number of rods varied from 2 to 10 . In total 1400 random meshes were created.

The meshes were generated on a computer with a Windows operating system then transferred to the Beowulf cluster to solve the generalized eigenvalue problem(a compiled MATLAB program).

The best 10 structures from each unit cell, which gave the largest gap-midgap ratio were optimised using the robust (but slow) Nelder Mead method [10] on the Condor system. The Nelder Mead algorithm was chosen for the optimisation 
because it only requires function evaluations and not derivatives which is ideal for our problem because the objective function has no calculable derivative. There are a number of constraints imposed on the variables mainly to ensure that it is possible to fabricate the proposed structure.

Each iteration is computationally intensive as it requires not only meshing the photonic crystal geometry but also solving the generalized eigenvalue problem.
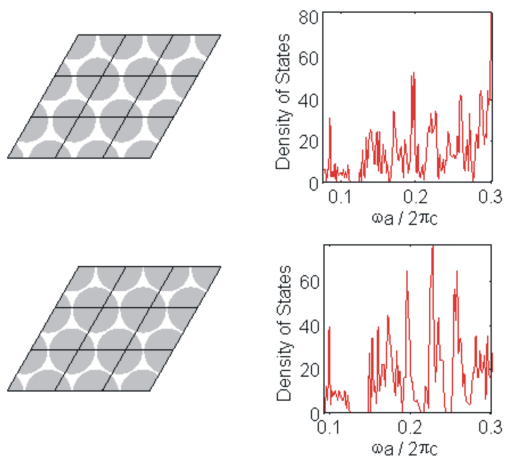

Fig. 2. Figure on the top left shows a tiled rhombic unit cell with a filling fraction of 0.753. On the top right is the density of states diagram for the unit cell. The band gap to mid-gap ratio is 0.118 . On the bottom left is the optimised tiled unit cell with a filling fraction of 0.838 . On the bottom right is the density of states for the optimised unit cell with a band gap to mid-gap ratio of 0.182 .

The results in this section were computed with an in house FEM code. The most improved mesh increased its gap-midgap ratio by $264.3 \%$ after optimisation. A density states graph is a concise method of visualising the results and is shown in Figure 2.

\section{Computer Costs}

In total 8940 meshes were created. Each generalized eigenvalue problem took approximately 6 minutes to run which equates to 37 days to compute the eigenvalues of each mesh if only one computer was used. When using the Beowulf cluster the workload was split up such that each processor would compute the eigenvalues for 100 meshes. However, the nodes used were dual processors so 200 meshes could be run on each node. As a result 45 separate jobs were submitted to our cluster using the EASY scheduler each taking approximately 10 hours to run. If the cluster was not being used at all all the jobs could have been run simultaneously in roughly 10 hours. However, the rate at which all the jobs are computed is dependent on how busy the cluster is. In our case, despite a heavily used cluster, it only took a few days to calculate the generalized eigenvalue problem from all the meshes. 
From the design of experiment 20 of the best PC geometries were optimised. The optimisation process was run for 12 hours for each structure. This equates to 10 days of computation on a single computer. Each of the 20 optimisation jobs were submitted to the Condor pool. With 20 free nodes, all the optimisation could be performed in only 12 hours. Once again, due to how busy the cluster was, a couple of days were required to complete all the jobs.

\section{Conclusion}

The randomisation proved effective in providing candidates for further analysis. Moreover, it confirmed the (known) good behaviour of the triangular lattice [4]. The Nelder Mead algorithm was successful in improving the good candidates.

The main contribution of the grid facilities was the reduction of about two months intense computation to a weekend. The scheduler on the Beowulf system was straightforward to use, but Condor had a shaper learning curve and less predictable completion times.

We have demonstrated the use of heterogeneous grid-enabled resources for an important industrial design process and in the future we hope that a widespread grid will enable the use of resources via brokering or pay-per-use and permit this sort of design to be a routine part of the process of developing new photonic devices.[4]

\section{References}

1. Axmann, W., Kuchment, P.: An efficient finite element method for computing spectra of photonic and acoustic band-gap materials - i. scalar case. Journal of Computational Physics 150 (1999) 468-481

2. Dobson, D.C.: An efficient method for band structure calculations in $2 \mathrm{~d}$ photonic crystals. Journal of Computational Physics 149 (1999) 363-376

3. K.S.Thomas, Cox, S., Beckett, D., Hiett, B., Generowicz, J., Daniell, G.: Eigenvalue spectrum estimation and photonic crystals. In: 7th International Europar Conference, Springer (2001) 578-586

4. Joannopoulos, J., Meade, R., Winn, J.: Photonic Crystals Molding the Flow of Light. Princeton University Press (1995)

5. University of Southampton: Research support services: Iridis (2002) http://www.iss.soton.ac.uk/research/iridis/.

6. University of Southampton: Research support services: Condor (2002) http://www.iss. soton.ac.uk/research/e-science/condor/.

7. Joe, B.: Geompack mesh generating software (2001) http://members.attcanada.ca/ bjoe/.

8. Cox, S.J., Dobson, D.C.: Maximizing band gaps in two-dimensional photonic crystals. Siam Journal on Applied Mathematics 59 (1999) 2108-2120

9. Cox, S.J., Dobson, D.C.: Band structure optimization of two-dimensional photonic crystals in h-polarization. Journal of Computational Physics 158 (2000) 214-224

10. Brent, R.: Algorithms for Minimization Without Derivitives. Englewood Cliffs, NJ:Prentice Hall (1973) 